\title{
XXIII. Magnetism and molecular rotation
}

\section{Lord Kelvin P.R.S.E.}

To cite this article: Lord Kelvin P.R.S.E. (1899) XXIII. Magnetism and molecular rotation, Philosophical Magazine Series 5, 48:291, 236-239, DOI: 10.1080/14786449908621326

To link to this article: http://dx.doi.org/10.1080/14786449908621326

$$
\text { 曲 Published online: } 08 \text { May } 2009 .
$$

Submit your article to this journal

LII Article views: 5

Q View related articles $\asymp$ 
If $v=\infty$, cases (a) and (b) cannot occur ; and in cases (c), (d) we see by ( 81 ) that $J=\infty$; that is to say, the whole onergy is carried away by the equivoluminal waves. If $v$ is very small in comparison with $u$, we find that although $J$ is infinite in cases $(a)$ and $(c)$, it is zero in cases $(b)$ and $(d)$. This to my mind utterly disproves my old hypothesis* of a very small velocity for irrotational wave-motion in the undulatory theory of light.

XXIII. Magnetism and Molecular Rotation. By Lord Kelvin, P.R.S.E. $\dagger$

\$1. CONSIDER the induction of an electric current in an endless wire when a magnetic field is generated around it. For simplicity, let the wire be circular and the diameter of its section very small in comparison with that of the ring. The time-integral of the electromotive force in the circuit is $2 \mathrm{AM}$, if $\mathrm{A}$ denote the area of the ring and $\mathrm{M}$ the component perpendicular to its plane, of the magnetic force coming into existence. This is true whatever be the shape of the ring, provided it is all in one plane. Now, adopting the idea of two electricities, vitreous and resinous, we must imagine an electric current of strength $\mathrm{C}$ to consist of currents of vitreous and resinous electricities in opposite directions, each of strength $\frac{1}{2} \mathrm{C}$. Hence the time-integrals of the opposite electromotive forces on units of the equal vitreous and resinous electricities are each equal to AM.

$\S 2$. Substitute now for our metal wire an endless tube of non-conducting matter, vitreously electrified, and filled with an incompressible non-conducting fluid, electrified with an equal quantity, $e$, of resinous electricity. The fluid and the containing tube will experience equal and opposite tangential forces, of each of which the time-integral of the line-integral round the whole circumference is $e \mathrm{AM}$, if the ring be a circle of radius $r$; and the effect of the generation of the magnetic field will be to cause the fluid and the ring to rotate in opposite directions with moments of momentum each equal to $e \mathrm{Al} r$, if neither fluid nor ring is acted on by any other force than that of the electromagnetic induction. Their angular velocities are therefore $e \mathrm{AM} / r w, e \mathrm{AM} / r w^{\prime}$, and their kinetic energies are $\frac{1}{2} e^{2} \mathrm{~A}^{2} \mathrm{M}^{2} / w, \frac{1}{2} e^{2} \mathrm{~A}^{2} \mathbf{M}^{2} / w^{\prime}$, where $w, w^{\prime}$ denote the masses of fluid and ring respectively.

$\S 3$. Suppose now for simplicity in the first place, the ring * "On the Reflexion and Refraction of Light," Phil. Mag. 1888, 2nd half year.

† Communicated by the Author; having been read before the Royal Society of Edinburgh, July 17, 1809 , 
to be embedded in ether, viewed as an incompressible solid, and attached to the ether in contact with it firmly enough to prevent slipping. The circuital impulse on the ring by the generation of the maguetic field will give rise to a rapidly subsiding train of waves of transverse vibration, of the kini which, in communications to Section A of the British Association* at its meeting in Bristol last September, I described as a solitary wave of the simplest possible kind in an elastic solid, and again, for periodic motion, as a very simple and symmetrical case of a train of periodic waves of transverse vibration. The work done by the circuital force on the riner is spent on waves of this class travelling outwards throngh ether, and in a very short time the ring comes practically to rest. It does not come to perfect rest suddenly by the departure from it of waves carrying away all its energy ; it. subsides to absolute rest in an infinite time according to the law $\epsilon^{-p t} \sin q t$. The resinously electrified fluid within the ring continues revolving with unaltered energy as long as the force of the magnetic field is maintained constant.

$\S 4$. The simple molecular arrangement thus imagined supplies the rotatory or revolutional motion, and the " moment of momentum," which, forty-three years ago, I pointed out $\dagger$ as wanted to explain, " simply by inertia and pressure," the rotation of the plane of polarization, then recently discovered by Faraday, for light transmitted through heavy glass in a powerful magnetic field along the lines of force. In my Baltimore I ectures I showed that embedded gyrostats would in fact produce exactly the rotation of the plane of polarizittion in a magnetic field discovered by Faraday. The idea which forms the subject of the present communication shows how the fly-wheels of the gyrostats may be started into rotation in virtue of the generation of the magnetic field and stopped when the magnetic field is annulled.

$\S 5$. The simply embedded gyrostat has not, however, the vibrational quality which is the essential of the StokesMaxwell $¥-$ Sellmeier vibratory molecule. For this a gyrostatic vibrator, capable of originating from a single blow on itselt a subsidential train of at least 200,000 waves of light, mort

* "On the Simplest Possible \&c.;" "On Continuity in Undulatory Theory, \&c.," B. A. Report, 1898.

+ "Dynamical lllustrations of the Magnetic and the Helicoidal Rotatory Effects of Transparent Bodies on Polarized Light."-Proc. R. S. L., vol. viii. June 1856 ; Phil. Mag. March 1857.

† See Rayleigh, Phil. Mag. July 1899, quoting from Camb. Liviv. Calendar, 1869.

Plil. Mag. S. 5. Vol. 48. No. 291. Aug. 1899. 


\section{Lord Kelvin on Magnetism and Moleculer Rotation.}

be connected with the surrounding ether by springs, baving sufficient resilience to store up in themselves the total energy thus radiated out. Taking now as gyrostat our electric doublet of vitreously electrified rigid hollow ring filled with fluid resinously electrified, consider what must be the nature of the elastic communication between it and a rigid lining of a spherical hollow in ether around it, to fulfil some of the known conditions of radiant molecules.

$\S 6(a)$. Let the spring connexion be equivalent to a simple force between I, the centre of inertia of ring and fluid, and 0 , the centre of the spherical sheath, varying directly as the distance between those points. The gyrostatic influence will be inoperati"e, and the result will be precisely the same as if we had a single Maxwell-Sellmeier material point at I, of mass equal to that of ring and fluid together.

$(b)$. Let points on the ring be connected by springs with points on the sheath. Supposing now the sheath to be held tixed, the stiffnesses and the tensions of these springs may be adjusted to give 21 arbitrary values for the coefficients in the quadratic for the potential energy of any infinitesimal displacement specified by three components of linear displacement of I, and three components of rotational displacement round axes through 1. The well-known solution of the problem of infinitesimal vibrations about a position of equilibrium of a rigid body, modified in respect to moments of inertia to take into account the fluidity of the incompressible fluid in the ring, gives us immediately the periods and geometrical specifications of six fundamental modes of simple harmonic vibration. Hence our combination, serving as a radiant molecule, without magnetic force, would give six bright lines (understood of course that each of the six periods is within the range of light-periods). Suppose now a vast number of such molecules, all equal and similar in every respect, but with different orientations, to be scattered through a flame. Each molecule, whatever its orientation, will give six lines of the same periods, though of different intensities when seen in any particular direction, according to the chances of orientation and of impulses. Hence each of the six bright lines will be perfectly sharp.

$\S 7$. Now suppose a magnetic field to be suddenly instituted. The moment of momentum generated in any one of the molecules is $r \mathrm{AM} \cos \theta$, where $\theta$ denotes the inclination of the axis of its ring to the lines of force. The gyrostatic influence will split each of our six fundamental modes of vibration into two, greater than it and less than it by equal very small 
differences. These differences will be different for different molecules, because of the different values of $\theta$ for their different orientations. Hence each bright line is not split into two sharp lines, but is broadenerl to an extreme breadth corresponding to the value $\theta=0$. No simplifying suppositions as to the character of the molecule, such as symmetry of forces and moments of inertia round the axis of the ring, can possibly give Zeeman's normal results of the splitting of a bright line into two sharp lines circularly polarized in opposite directions, when the light is viewed from a direction parallel to the lines of magnetic force; and the dividing of each bright line into three, each plane-polarized, when the light is viewed from a direction perpendicular to the lines of force. Hence, although from 1856 till quite lately I felt satisfied in knowing that it sufficed to explain Faraday's magneto-optic discovery, I now, in the light of Zeeman's recent discovery, discard my old tempting gyrostatic hypothesis for an irrefragable reason, which is virtually the same as that stated by Larmor* in the following words :- "Hence a principal oscillator magnetically tripled must be capable of being excited with reference to any axis in the molecule; otherwise there would be merely hazy broadening or duplication instead of definite triplication."

$\S 8$. It now seems to me that the theory of $H$. A. Lorentz (of Leyden), as expressed by equations (1) in Zeeman's first paper " On the Influence of Magnetism on the Nature of the Light emitted by a Substance" $\dagger$, is essentially true.

$\S 9$. Though it cannot explain Zeeman's discovery, the molecular rotation cansed by the institution of a magnetic field, which is the subject of the present conmunication, may, however, be considered as interesting not only becanse the idea of it seems to be new in electromagnetic theory, but also because it may conceivably constitute the explanation of Faraday's diamagnetism. Go back to $\S \S 2,3$ above, and remark that if a body containing a vast number of the molecules there described is situated between the poles of a steel magnet, the total energy will be greater than if there were nothing but ether between the poles, by a difference equal to the kinetic energy of the motion of the resinuously electrified fluid. Hence if a body containing the supposed congregation of molecules is movable, it must be repelled from the place of strong magnetic force between the poles to places of weaker force further from them.

- Phil. Mag. vol. xliv. 1897, "On the Theory of the Magnetic Influence on Spectra," p. 507.

† Phil. Mag. vol. xliii. (1897) p. 226. 